\title{
Foreword
}

\section{Geriatric Anesthesia: Can We Achieve the Goal of Returning our Elderly to Baseline or Improved Function?}

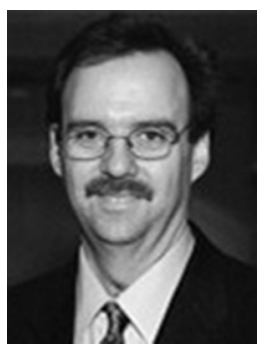

Lee A. Fleisher, MD, FACC, FAHA Consulting Editor

The importance of age on mortality has been known for decades. It is well known that comorbidities increase with age, but there is great variability between adults of the same age. We all know of the frail elderly who requires all of our talents to "get them through the operation" as opposed to the healthy individual who is still playing three sets of tennis. Yet, recent research has clearly demonstrated that there is significant organ dysfunction postoperatively, including postoperative cognitive changes in this group. In addition, recent research incorporating postdischarge (30-day and 1-year) mortality has demonstrated that the actual rate of death is much higher than was traditionally thought. It is for this reason that the current issue of Anesthesiology Clinics was commissioned. It includes a diverse group of articles that span the entire perioperative period and includes chronic pain issues. It also includes a surgeon's perspective since care of the elderly is clearly a team sport. Finally, we should recognize that surgery may not always be the optimal patient-oriented care plan, and palliation should be considered. Therefore, this issue should serve as a resource for all of us who provide care for the elderly.

In order to create such an issue, I was able to enlist two outstanding young investigators to be the coeditors. Mark Neuman, MD, MSc trained in anesthesiology and completed the Robert Wood Johnson Clinical Scholars Program. He is currently Assistant Professor of Anesthesiology and Critical Care at the Perelman School of Medicine at the University of Pennsylvania with joint appointments as an Assistant Professor of Medicine (Geriatrics) and Senior Fellow in Penn's Leonard Davis Institute of Health Economics. He has recently been awarded a major, multicenter pragmatic trial of 
spinal versus general anesthesia from the Patient Centered Outcomes Research Institute and is the 2015 ASA Presidential Scholar. Charles Brown, MD completed residencies in both anesthesiology and emergency medicine. He is Assistant Professor of Anesthesiology and Critical Care Medicine at the Johns Hopkins School of Medicine. He received a Research Career Development Core Award from the NIH-funded Johns Hopkins Claude D. Pepper Older Americans Independence Center. His research interests include quality and outcomes regarding perioperative management of older adults, specifically focused on postoperative delirium, cognitive change, and transfusion practices.

Lee A. Fleisher, MD, FACC, FAHA Perelman School of Medicine at the University of Pennsylvania

Philadelphia, PA 19104, USA 